\title{
Single-Ion Implantation for the Development of Si-Based MOSFET Devices with Quantum Functionalities
}

\author{
Jeffrey C. McCallum, David N. Jamieson, Changyi Yang, Andrew D. Alves, Brett C. Johnson, \\ Toby Hopf, Samuel C. Thompson, and Jessica A. van Donkelaar
}

Centre of Excellence for Quantum Computation and Communication Technology, School of Physics, University of Melbourne, Melbourne, VIC 3010, Australia

Correspondence should be addressed to Jeffrey C. McCallum, jeffreym@unimelb.edu.au

Received 4 August 2011; Accepted 29 August 2011

Academic Editor: Robert G. Elliman

Copyright ( $\odot 2012$ Jeffrey C. McCallum et al. This is an open access article distributed under the Creative Commons Attribution License, which permits unrestricted use, distribution, and reproduction in any medium, provided the original work is properly cited.

Interest in single-ion implantation is driven in part by research into development of solid-state devices that exhibit quantum behaviour in their electronic or optical characteristics. Here, we provide an overview of international research work on single ion implantation and single ion detection for development of electronic devices for quantum computing. The scope of international research into single ion implantation is presented in the context of our own research in the Centre for Quantum Computation and Communication Technology in Australia. Various single ion detection schemes are presented, and limitations on dopant placement accuracy due to ion straggling are discussed together with pathways for scale-up to multiple quantum devices on the one chip. Possible future directions for ion implantation in quantum computing and communications are also discussed.

\section{Introduction}

Interest in single-ion implantation is motivated in part by the desire to be able to produce electronic and optical devices in which the characteristics of the device are governed by the properties of individual atoms or defects and by extension for computational operations to be performed through controlled coupling of these atoms or defects, formed into arrays or lines, using interactions dictated by the laws of quantum mechanics $[1,2]$. Some of the impetus for research into development of quantum computing derives from an address to the American Physical Society on nanotechnology given by Feynman in 1959 and a later address on simulating physics with computers $[3,4]$. Considerable time past before the necessary fabrication and measurement infrastructure, and theoretical understanding was available to even approach the early vision articulated by Feynman.

In 1998, Kane proposed an architecture for a quantum computer based on encoding information on the donor nuclei of individual phosphorus atoms embedded in a silicon matrix [5]. This provided a plausible pathway to realisation of a solid-state quantum computer and prompted interna- tional attention to be focussed on developing the necessary expertise and infrastructure to allow such a device to be fabricated. Interest in single-ion implantation and singleion detection has grown as part of this research effort. After the Kane proposal, a range of other architectures and encoding mechanisms for quantum bits, qubits, were proposed [68]. Researchers also realised that the Kane architecture suffered from some major technical problems including spatial oscillations in the exchange coupling strength and crosstalk in gate control prompting further improvements in architectures to be put forward $[9,10]$. The key importance of being able to initialise and read out qubits also prompted research into development of electron reservoirs that could be manipulated through suitable variation of the electrostatic landscape to allow electrons to be loaded onto or removed from a nearby qubit in a precisely controlled manner. Gate-defined quantum dots have proved particularly useful in this regard and recent advances in single electron manipulation and spin readout have relied on this key breakthrough [11-14].

As device dimensions continue to shrink, counted implantation of ions and their controlled placement are also of interest for deterministic doping of conventional 


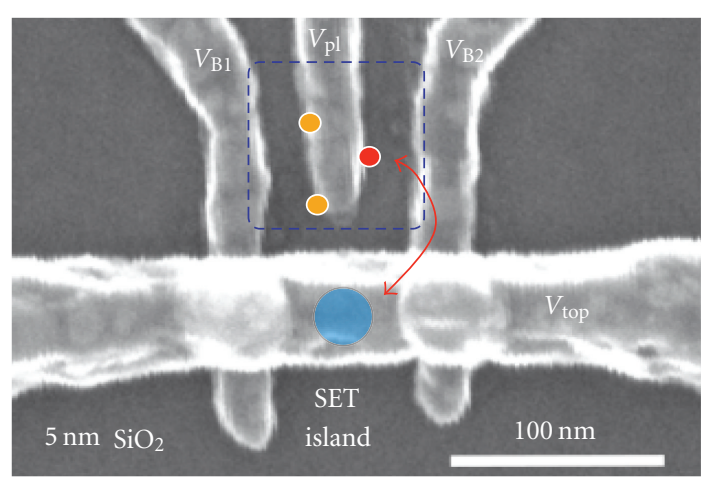

(a)

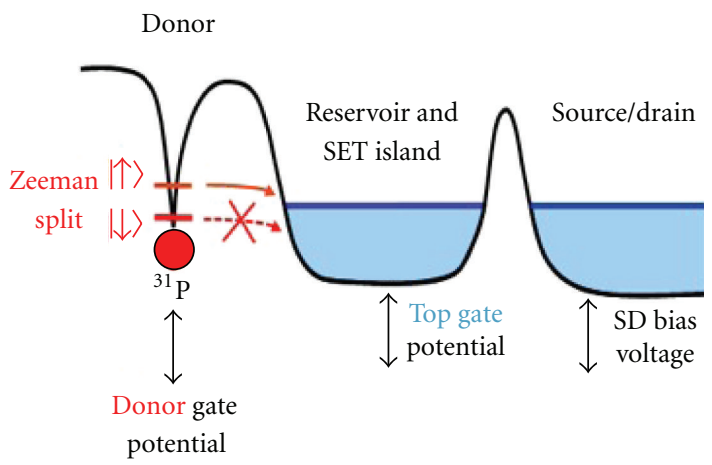

(b)

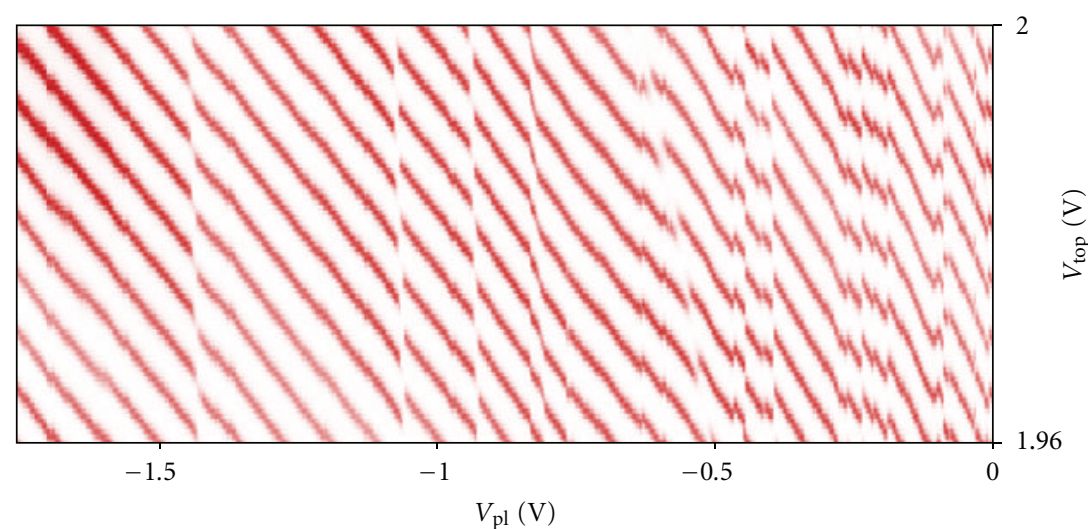

(c)

FIGURE 1: Silicon SET with a small number of phosphorus atoms implanted nearby. (a) SEM image of the device. (b) In the presence of a large externally applied magnetic field tunneling from a phosphorus donor to the SET island becomes spin dependent. (c) SET current versus top and plunger gate voltage. The breaks are due to charge transfer events (as in [14]).

metal-oxide field effect transistors (MOSFETs). The aim of deterministic doping is to remedy the variation in threshold voltage shift and off-state current that occurs due to statistical fluctuations in the number and distribution of implanted dopants [15-20]. Workshops on deterministic doping have been held as part of the International Technology Roadmap on Semiconductors Emerging Research Materials program but there is a large gap between what is achievable in research devices in the laboratory and what would be required for scale up to an industrial process, and the pathways for achieving scale-up are far from being clear at this stage [21].

Single-ion implantation is also of interest for controlled placement of individual optical centres in a suitable host matrix for development of single photon sources and other optical elements for use in optical quantum computing and quantum communications. Considerable progress has been made, for example, on engineered placement of nitrogenvacancy centres in diamond [22-26]. Interest in measurement and manipulation of individual optical centres grows as research into quantum communications intensifies.

Single-ion implantation of optical centres is not the focus of this paper, instead the emphasis is on single-ion implantation and single-ion detection for development of electronic devices for quantum computing. The scope of international research into single-ion implantation is presented in the context of our own research in the Centre for Quantum Computation and Communication Technology in Australia. The paper begins with a brief discussion of a phosphorus in silicon quantum computing device in which single-shot readout of electron spin state has recently been demonstrated [14]. This device allows many of the important features of silicon quantum device design and construction to be understood. Single-ion detection via ion-beam-induced current (IBIC) is then introduced, and the attributes and challenges of this method are discussed including proposed future developments. Single-ion detection through measurement of changes in the source-drain current in MOSFET devices is an important alternative methodology that is suitable for some classes of devices, and research on this approach is also presented.

\section{Single-Ion Detection: Ion-Beam-Induced Charge}

Figure 1(a) shows a scanning electron microscope (SEM) image of a gate-defined quantum dot structure or silicon single electron transistor (SiSET) with a small number of phosphorus atoms implanted within the region bounded by the dashed line $[14,27]$. The SiSET is formed using $\mathrm{Al}$ gates deposited on a high-quality $5 \mathrm{~nm} \mathrm{\textrm {SiO } _ { 2 }}$ thermal 
oxide grown on a high-purity Si substrate. A small reservoir of electrons is formed on the central SET island through appropriate choices of top-gate $\left(V_{\text {top }}\right)$ and barrier-gate $\left(V_{B 1}\right.$, $V_{B 2}$ ) potentials. The electrons on the SET island are tunnelcoupled to the source and drain reservoirs. With appropriate adjustment of the electrostatic landscape, an electron can also tunnel onto the island from a nearby charge centre such as an implanted phosphorus donor. This can be achieved using the plunger gate $\left(V_{\mathrm{pl}}\right)$. Transfer of an electron from the charge centre to the island has a dramatic effect on the source-drain current, so the tunnelling event can readily be detected.

In the presence of a large externally applied magnetic field tunnelling between the charge centre and the island becomes spin dependent, as illustrated in Figure 1(b). Figure 1(c) shows the variation in the SET current as a function of the voltages on the top and plunger gates. The breaks are due to charge transfer events. Since the tunnelling probability is very sensitive to the distance between the charge centres and the island, different regions of the bias-spectroscopy diagram correspond to tunnelling events from different charge centres [14, 27]. For this particular device, timed implants were used to achieve 3-5 implanted phosphorus atoms within a $90 \times 90 \mathrm{~nm}^{2}$ aperture in the polymethyl methacrylate (PMMA) mask opened adjacent to the SET island (dashed region in Figure 1(a)). Recent measurements positively identify phosphorus as one of the charge centres based on observation of the hyperfine splitting. A double-gated nanoscale field-effect transistor, with a small number of phosphorus donors implanted into the conduction channel, has also been fabricated to study electron transport through individual phosphorus atoms [13].

Detected single-ion implantation allows precise control over the number of ions implanted through an aperture. Detection of single-ion impacts can be achieved in a number of different ways including detection of secondary electron emission from the surface [28], observation of ion-track formation in some suitable resist coated onto the surface [29], detection of changes in the source-drain current of a MOSFET device [18], or detection of the electron-hole pairs formed as the ions lose energy through electronic interactions with the substrate [30]. This latter method has the advantage that detection of the energy deposited by the ions in the lattice allows real-time differentiation between events where the ions stop in the substrate and those where they stop in an overlying resist or oxide layer or some other undesired location. Devices for quantum computation generally require implantation of low-energy ions due to the need for close proximity to surface gates. The detection of sub- $20 \mathrm{keV}$ ions is somewhat challenging because relatively few electron-hole pairs are produced by each ion. With care in device fabrication and packaging to reduce capacitance and through use of low-noise electronics it is possible to routinely and reliably detect implantation of the low-energy ions required for quantum computing devices.

The detection method is illustrated in Figure 2. The detector is a reverse biased PIN structure fabricated in a highpurity Si substrate. Two surface aluminium electrodes contact the boron-doped p-wells. The back contact consists of an

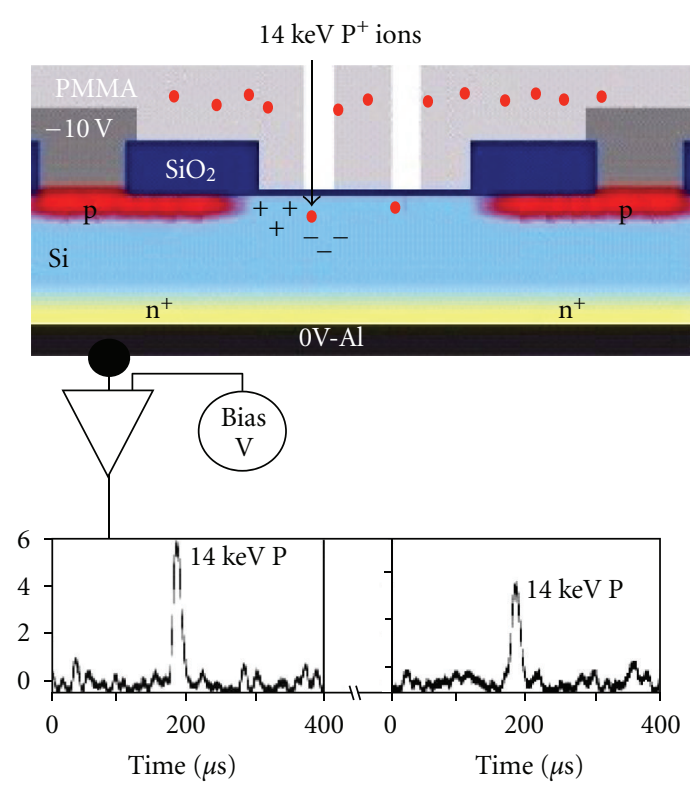

Figure 2: Schematic of the PIN single-ion detection system integrated into the high-purity $\mathrm{Si}$ substrate. The surface detector electrodes are connected to the p-wells on each side of the $5 \mathrm{~nm}$ thick gate oxide which has a width of $10 \mu \mathrm{m}$. Apertures in the PMMA mask allow ions to enter the substrate in selected areas. Two transients detected in the external circuit from single-ion impacts are shown in the bottom panel of the figure (as in [30]).

n-type phosphorus diffused layer and $\mathrm{Al}$ contact. Detection of the transient signal due to ion impacts is achieved by connecting the detector electrodes to a cooled MOXTEK four-terminal junction field effect transistor (JFET) with an integrated transistor resetting circuit. An external preamplifier module controls the JFET. Each implanted ion produces approximately 1000 electron-hole pairs which drift in the internal electric field and induce the charge transient detected by the external circuitry. $14 \mathrm{keV}^{31} \mathrm{P}^{+}$ions have a projected range of about $22 \mathrm{~nm}$ when implanted into $\mathrm{Si}$ through a $5 \mathrm{~nm} \mathrm{SiO} \mathrm{Si}_{2}$ surface layer, as predicted by the modelling code SRIM [31]. Only $34 \%$ of the initial kinetic energy produces ionization events hence the need for optimised design of the detection system so that the noise threshold is as low as possible. Other details of the detection system can be found in [30, 32, 33].

Ion-bean-induced charge (IBIC) measurements using high energy ions is a very useful tool for studying detector efficiency and optimising detector design prior to attempting a keV single-ion implantation experiment. The righthand panels of Figure 3 show IBIC maps of a quantum computing device collected with a scanned $500 \mathrm{keV}$ He microbeam from the Melbourne Pelletron. The top panel is a large area scan, while the bottom panel is a closeup of the site where the single-ion implantation will take place, showing the desired high charge collection efficiency in this region. The two left hand panels are the corresponding SEM images of the device. The purple regions of moderately high charge collection efficiency arise from a diffused channel stopper that is incorporated into the device design to prevent crosstalk 


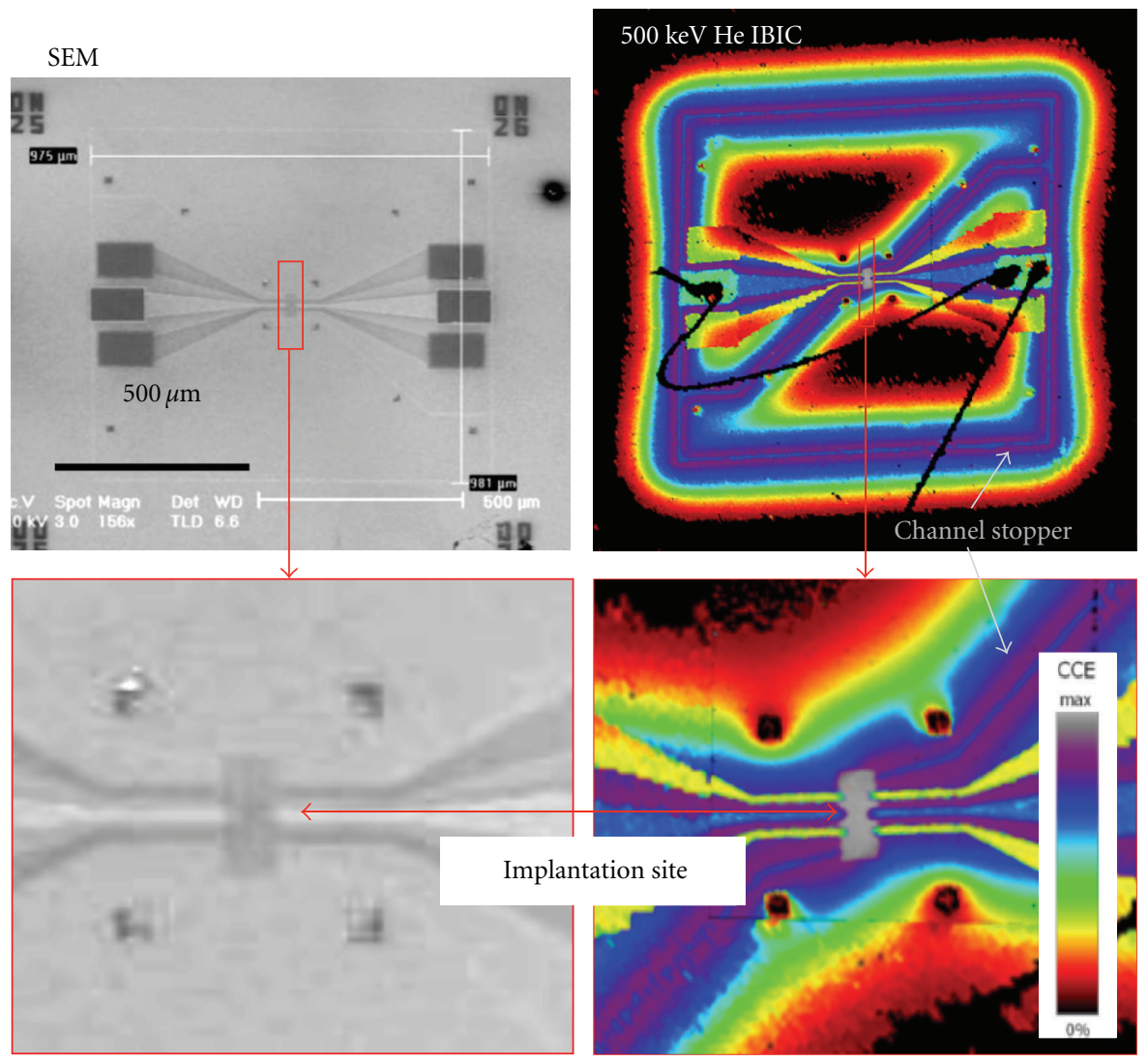

FIGURE 3: IBIC maps of a quantum computing device collected with a scanned $500 \mathrm{keV}$ He microbeam. The top right-hand panel is a large area scan while the bottom right-hand panel is a close up showing the desired high charge collection efficiency in the site where the single-ion implantation will take place. The two left-hand panels are the corresponding SEM images of the device.

between device components. The IBIC maps show that the channel stopper is inadvertently coupled to the detector leads increasing the capacitance. This issue is being addressed in later generations of devices and highlights the importance of IBIC with $\mathrm{MeV}$ ions in allowing such issues to be identified.

\section{Single-Ion Implantation Challenges}

Some of the key challenges facing researchers in developing single-ion implantation for quantum computing are limitations on the accuracy of placement of implanted ions due in part to ion straggling, limits on the ability to detect ion implantation events above the noise floor as the ion energy is reduced due to the decrease in the fraction of the ion energy that contributes to ionisation events, and the need to develop appropriate pathways for scale-up so that multiple implant sites and devices can be implanted sequentially.

The variation in ion range and longitudinal straggling with ion energy is illustrated in Figure 4(a) for keV P and Sb ions. Sb implanted quantum devices are being investigated by Schenkel and coworkers $[34,35]$. For $14 \mathrm{keV}$ P, the projected range is $\sim 22 \mathrm{~nm}$, and the longitudinal range straggling gives rise to a variation in depth of $\sim \pm 11 \mathrm{~nm}$. The corresponding lateral range straggling is $\sim 8 \mathrm{~nm}$. The situation is better for $\mathrm{Sb}$ as can be seen in Figure 4(a). However, ${ }^{121} \mathrm{Sb}$ has a nuclear spin of $I=5 / 2$ [36], giving rise to extra spin states compared to the simple two-state system for P $(I=$ $1 / 2$ ), and the heavier mass of the Sb ions is less compatible with implantation through a preexisting thermally grown gate oxide since the damage is likely to be more difficult to repair than is the case for $\mathrm{P}$ [37]. Indeed, Lo et al. adopted a process where they grew the oxide after the $\mathrm{Sb}$ implantation, [36] relying on the relatively small intrinsic diffusivity of $\mathrm{Sb}$ at the oxide growth temperature, but this approach also adds to the uncertainty in how well alignment with surface gates can be achieved. The effect of ion straggling on the uncertainty in the placement of $\mathrm{P}^{+}$ions is further illustrated in Figure 4(b) which shows the predicted probability distributions for the implantation of $10000014 \mathrm{keV} \mathrm{P}^{+}$ions through three circular apertures $10 \mathrm{~nm}$ (top panel) and $20 \mathrm{~nm}$ (bottom panel) in diameter [38]. In each case the aperture centres are spaced $30 \mathrm{~nm}$ apart. The ion impacts were randomly distributed over the apertures. The lateral and longitudinal range contribute significantly to the overall uncertainty in location of the implanted ions.

The system of three donors at adjacent implant sites $30 \mathrm{~nm}$ apart depicted in Figure 4(b) is a prototype model to test whether ion implantation could reasonably be used to produce a device that would allow demonstration of quantum transport via the coherent tunnelling adiabatic 


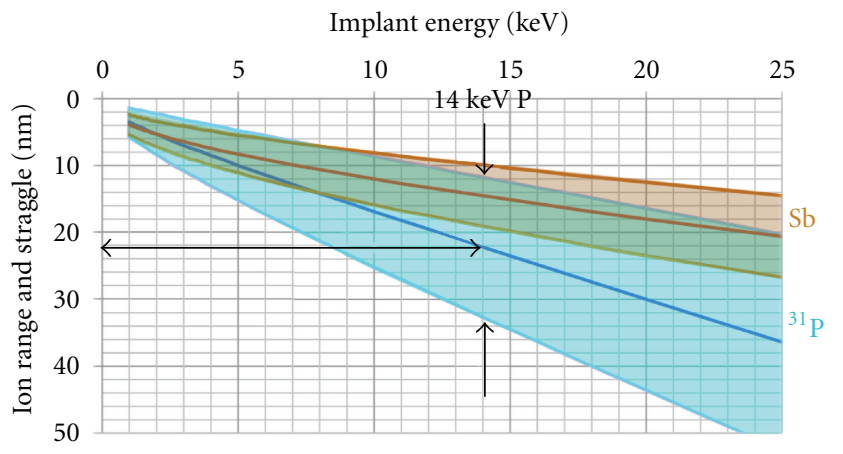

(a)

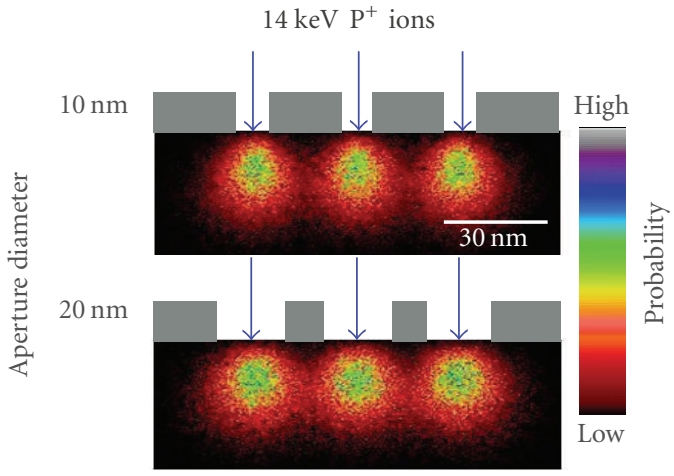

(b)

Figure 4: The effect of ion range straggling on the uncertainty in location of keV P and Sb ions implanted into Si: (a) variation in ion range and longitudinal straggling with ion energy for keV P and Sb ions. (b) Probability distributions for the implantation of $10000014 \mathrm{keV}$ $\mathrm{P}^{+}$ions through three circular apertures spaced $30 \mathrm{~nm}$ centre to centre. The aperture diameter is $10 \mathrm{~nm}$ in the top panel and $20 \mathrm{~nm}$ in the bottom panel.

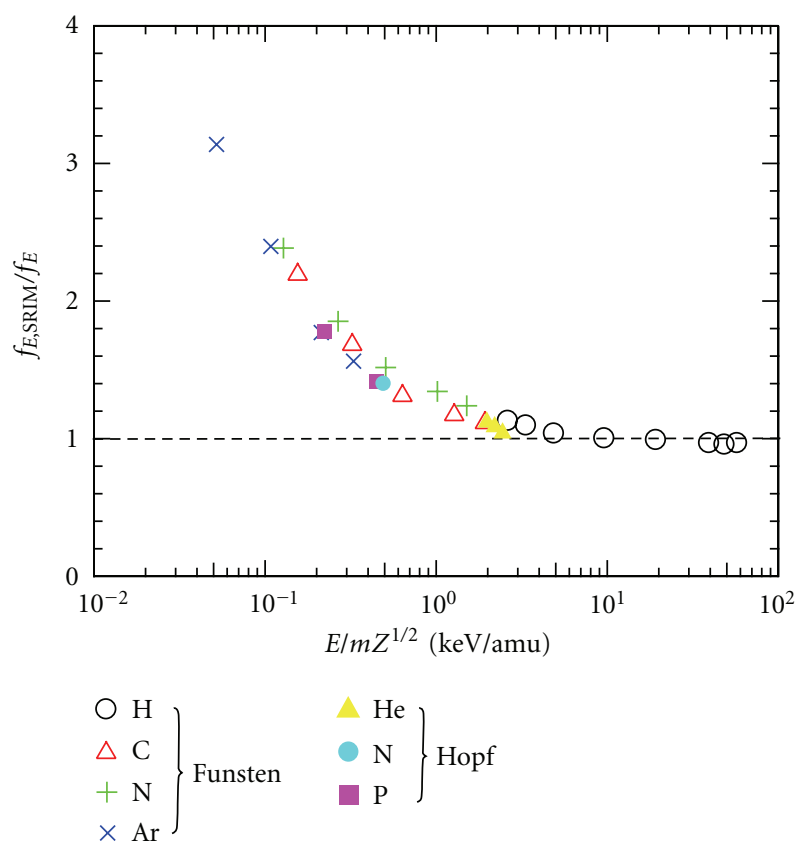

Figure 5: The fraction of an ions initial energy lost to electronic stopping processes by both the primary ion and recoils as predicted by SRIM $\left(f_{E, \text { SRIM }}\right)$ divided by the value obtained from measurement using our single-ion detection system $\left(f_{E}\right)$ and compared with previous data of Funsten et al. (as in $[39,40]$ ).

passage (CTAP) protocol [42]. CTAP is a protocol for the spatial transport of a quantum state between two points on a quantum chain. Quantum transport is a necessary component of distributed quantum computing, and the CTAP protocol has some advantages over competing transport mechanisms [38]. Within the limits of the approximations used, simulations show that one in six three-donor devices produced by $14 \mathrm{keV} \mathrm{P}$ implantation could be suitable for CTAP; while a reduction of the implantation energy to $7 \mathrm{keV}$ is predicted to yield one in two working devices. More sophisticated modelling using large-scale atomistic tightbinding simulations has revealed that donor displacements along the direction of the donor chain, as opposed to those perpendicular to it, have a larger impact on whether the protocol will work or not but that some retuning of the gate potentials may be possible to compensate [43].

The examples presented here are all for donor-based quantum computing devices but it is worth noting that acceptor-based quantum computing is also a possibility. It has been shown in lightly boron-doped silicon samples that transitions between the acceptor energy levels can be induced by an applied resonant AC electric field and that Stark tuning of level spacing can be achieved with an external DC electric field [44]. However, the authors also noted a strong sensitivity of the system to random local strain variation, and this may be problematic for boron qubits formed by ion implantation.

While ion straggling can be reduced by reducing the ion energy, the projected range of the ions still needs to be large enough to place the ions deeper than the extent of any gate oxide or other overlayers that may be present. Also, donors placed too close to the surface may hybridize with states at the oxide interface which may not be desired $[45,46]$. As the ion energy decreases, the fraction of the energy that is available for ionisation events also reduces adding to the difficulty of single-ion detection via electron-hole pairs. Also, the electronic stopping predicted by SRIM has been shown to be in error in the low-energy regime $[32,39,40,47$, 48]. SRIM overestimates the electronic stopping as illustrated in Figure 5 which shows the fraction of an ions initial energy lost to electronic stopping processes by both the primary ion and recoils as predicted by SRIM $\left(f_{E, S R I M}\right)$ divided by the value obtained from measurements using our singleion detection system $\left(f_{E}\right)$ or from previous measurements of Funsten et al. [39, 40]. Good agreement is obtained despite the fact that the detection systems are quite different in the two cases. In the work of Funsten et al. the mean energy lost to electronic processes by incident ions, and recoils was found through measurement of the change in photocurrent 
in silicon photodiodes under ion bombardment. Large area detectors were used and relatively high fluences could be tolerated before the detectors would degrade. In our singleion detectors the ionisation response is measured in a central region only $10 \times 10 \mu \mathrm{m}^{2}$ where the ions can penetrate through the thin $5 \mathrm{~nm}$ oxide. The ionisation is directly measured for individual ions, and the energy spectrum is collected for multiple ions but only relatively small numbers of ions can be detected before the accumulated damage reduces the detection efficiency. Only a few hundred ions can be detected before a new detector is needed. Recently Akkerman and Barak have developed a model for the energy partitioning between electronic and nuclear components for low-energy ions in silicon, and this model is found to provide a good fit to the data of Funsten et al. and to our own data $[40,49,50]$.

To compensate for the decreasing number of electronhole pairs produced with decreasing ion energy one can look for more sensitive detection systems. Avalanche photodiodes (APD) can be used to detect single-electron hole pairs generated by single photons [51]. This approach has recently been adapted for single-ion detection $[52,53]$. The avalanche diodes are operated in Geiger mode by biasing past reverse breakdown for a short-time period during which the detector is sensitive to a single electron or hole injected into the junction. The high fields produce an avalanche cascade leading to a Geiger signal. Near 100\% detection efficiency has been demonstrated for $250 \mathrm{keV} \mathrm{H}^{+}$ions [53]. In the current detector design thick surface layers and structures prevent low-energy ions from reaching the active region of the device but the methodology has considerable merit, and there is scope for further development.

Scale-up to multiple qubits and transport structures on the same device requires some method to ensure registration of the ions to the aperture array, that is, that one and only one ion is implanted through each aperture in the lithographic mask. It is impractical to use multiple lithographic steps with only one aperture open at each step. Ideally if a method could be devised to have a nanometer diameter ion beam and nanometer-level accuracy in the position of the beam with respect to a substrate, it would be possible to form qubit arrays without a lithographic mask. Proposals exist for ion-trap-based ion sources that could deliver arrays of single ions with nanometre spatial precision but these systems are still in development [54-56]. A focussed ion beam (FIB) system can produce a sub-10 nm diameter beam, and this is the approach used by Shinada et al. to produce ordered dopant arrays in the channel of a back-gated silicon on insulator device [15]. The single-ion impacts were detected by measuring the change in the source drain current of the device during implantation. Although the device was rather large, having channel width and length of $300 \mathrm{~nm}$ and $3.2 \mu \mathrm{m}$, respectively, theoretically single-ion implantation could be achieved in nanoscale devices via this approach, however, no beam halo can be tolerated, and this will be very difficult to achieve. An alternative approach is to use a lithographically defined mask on the sample combined with a scanned nanoaperture used to collimate the ion beam and which can be positioned with nanoscale accuracy. This is the approach adopted by Schenkel and coworkers and by our group in Melbourne [38, 57, 58].

Figure 6(a) illustrates the concept of using a scanned nanoaperture to sequentially implant ions through an array of nanoapertures lithographically patterned on a substrate. Here, the nanoaperture is a slot in a cantilever. The position of the scanned aperture can be registered to the substrate by detecting ions implanted into a registration zone. The aperture can then be repositioned to the implant zone to perform the deterministic implants. Figure 6(b) shows the apparatus used in these experiments. Nanopositioning is achieved with a three-axis Attocube stack [59]. The singleion detector can be cooled for low-noise operation. An SEM micrograph of one of the slot nanoapertures is shown in Figure 6(c). The slot aperture is formed by FIB milling of a Si cantilever [38]. The slot is backfilled by in situ deposition of $\mathrm{Pt}$ dissociated from a precursor gas by the ion beam to achieve the desired aperture width. A $60 \mathrm{~nm}$ slot is shown here. The narrowest uniform slot width we have achieved to date is $30 \mathrm{~nm}$. Figure 6(c) shows a series of irradiation lines in PMMA formed by $14 \mathrm{keV}$ Ar ion irradiation through the $60 \mathrm{~nm}$ wide aperture and stepping of the aperture using the nanopositioner. We are currently working towards full testing of our single-ion detection system integrated with the nanoaperture positioning system.

Finally in this section it is worth mentioning an alternative technology that can place dopant atoms with nearatomic precision. Hydrogen-resist lithography uses a $\mathrm{H}$ terminated $\mathrm{Si}(001)$ surface and selective removal of $\mathrm{H}$ via a scanning tunnelling microscope tip followed by controlled adsorption of $\mathrm{PH}_{3}$ molecules at the exposed sites to incorporate $\mathrm{P}$ atoms into $\mathrm{Si}$ with near-atomic control over the position [60]. Remarkable progress has been made using this technology and recently a nanoscale Si quantum dot device with approximately six $\mathrm{P}$ atoms in the quantum dot has been demonstrated [61]. The device features in-plane sourcedrain leads and gates also formed using $\mathrm{H}$-resist lithography and $\mathrm{P}$ incorporation. This technology does not suffer from ion straggling and has the potential to produce qubits and transport arrays with appropriate positioning accuracy but it does require quite painstaking work in its continued development. In the longer term, ion implantation may have a significant role in fabrication of quantum optical devices where atoms and optical defects are coupled to optical cavities and waveguide structures [62]. In these structures, restrictions on the placement accuracy of atoms or optical defects are likely to be more relaxed.

\section{Single-Ion Detection: Drain Current Modulation}

In prefabricated nano-MOSFET devices where it is not possible to incorporate the previously mentioned single-ion detection system it can still be possible to perform realtime single-ion detection by monitoring the changes in the source-drain current of the device due to ion damage. This was the approach used by Shinada et al. in their back-gated silicon on insulator MOSFET device [15]. The approach has also been used by Batra et al. $[63,64]$. Our own work in 


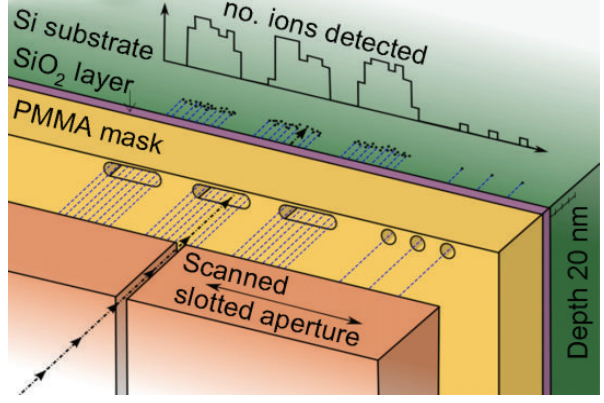

(a)

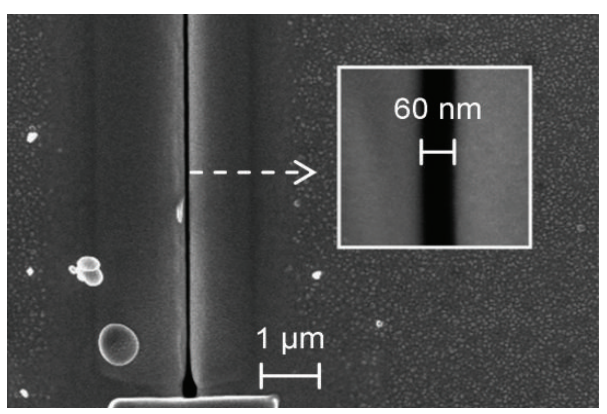

(c)

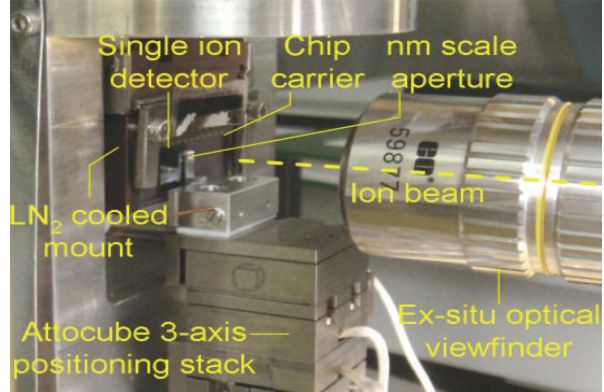

(b)

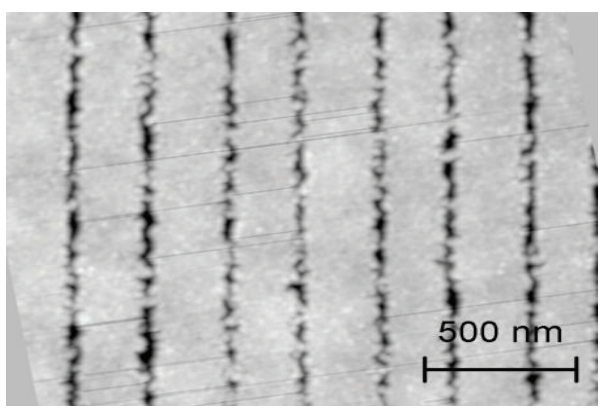

(d)

Figure 6: Ion registration with a lithographically patterned substrate using a scanned nanoaperture: (a) illustration of the concept of using a scanned nanoaperture to sequentially implant ions through an array of nanoapertures patterned on a substrate. (b) The nanopositioning system integrated with the single-ion detection system. (c) A $60 \mathrm{~nm}$ wide slot aperture in a Si cantilever formed by FIB milling and Pt back-filling. (d) A series of irradiation stripes formed in PMMA by $14 \mathrm{keV}$ Ar irradiation through the slot aperture.

this area and that of other researchers is motivated by recent observations of quantum transport through individual randomly distributed dopant atoms fortuitously located at appropriate positions in the channels of nanoscale MOSFETs to affect the conduction $[20,65]$. Single-ion implantation offers the possibility of deliberately placing dopants in the channel to produce quantum effects.

We have performed single-ion implantation studies on nanoscale silicon-on-insulator (SOI) MOSFETs using $500 \mathrm{keV} \mathrm{He}^{+}$and $14 \mathrm{keV} \mathrm{P}^{+}$ions and observed changes in the drain current due to the ion impacts [41]. Two different finFET device structures were used in the study as shown in Figure 7. In the case of the $500 \mathrm{keV} \mathrm{He}$ irradiations the particle range was sufficient for the ions to pass right through the wrap-around gate and the channel and the buried oxide (BOX) and stop in the substrate. As shown in Figure 7(a), in this case discrete upward steps were observed in the drain current, $I_{D}$, consistent with the expected rate of ion arrival. This was accompanied by a negative shift of the IV curve which is consistent with trapped charge being created in the BOX. For $14 \mathrm{keV}^{+}$ions a different device geometry with a split-gate design was used so that the low-energy ions could reach the channel. Now, downward steps in $I_{D}$ occurred, as shown in Figure 7(b), and a corresponding positive shift of the IV curve was observed consistent with negatively charged interface states being generated. The decrease in $I_{D}$ was consistent with introduction of Frenkel pairs into the channel.

Subsequent experiments have shown that it is difficult to unambiguously identify low-energy single-ion impacts in these devices on a routine basis. In some cases no variation in the drain current is detected above the noise. Device modelling with the technology computer aided design (TCAD) software package may go part way to explaining this [66]. Figure 8 shows the expected current density in the split gate device. The current density is greatest under the gates where the low-energy ions cannot penetrate which may reduce the sensitivity of the devices to low-energy ion impacts. These devices offer the possibility of back-gating as was used by Shinada et al. [15] in their deterministic doping studies. We are currently investigating this avenue for improved sensitivity to low-energy ion impacts.

\section{Conclusions}

Single-ion implantation has contributed to rapid prototyping of devices for solid-state quantum computer development. Phosphorus implanted quantum devices exhibiting spin-dependent transport and single shot spin readout have been demonstrated. Single-ion detection schemes allow individual ions to be implanted into lithographically patterned device structures with high precision but ion range straggling places fundamental limits on the positioning accuracy and in the longer-term ion implantation may not be able to compete with the hydrogen-resist lithography approach which can produce quantum devices with near-atomic precision in dopant placement. Ion range straggling can be reduced by decreasing the ion energy, but surface proximity effects can become a problem, and there are a diminishing number 

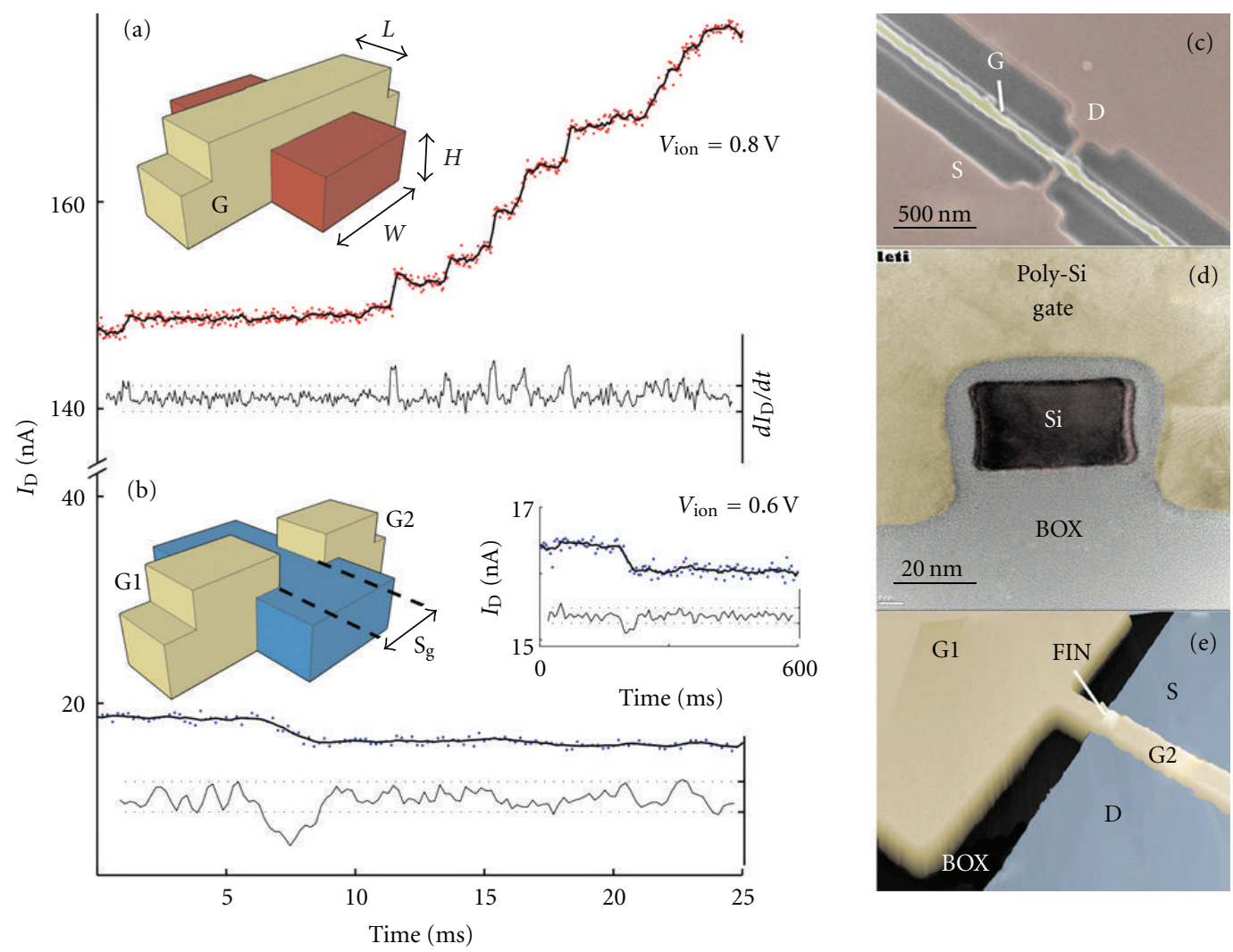

FIgure 7: Changes in $I_{D}$ in nanoscale MOSFETs during (a) $500 \mathrm{keV} \mathrm{He}^{+}$and (b) $14 \mathrm{keV} \mathrm{P}^{+}$ion irradiation. Discrete steps represent singleion impacts. Schematics of the devices are shown as insets. False color scanning electron microscopy, transmission electron microscopy, and atomic force microscopy images of a double gated MOSFET identical to those under study are shown in (c), (d), and (e), respectively (as in $[41])$.

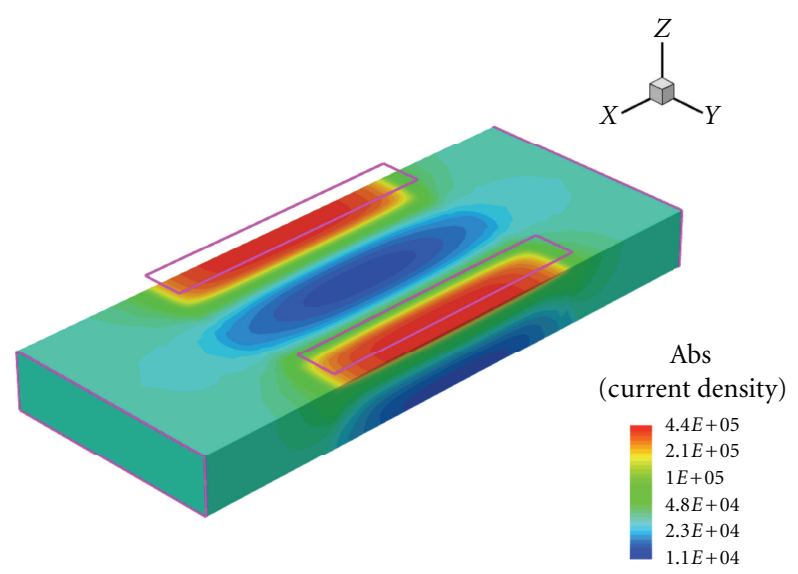

FIgURe 8: TCAD modelling of current density in the split-gate finFET device, showing the low current density in the exposed sections of the device where ion impacts will occur.

of electron-hole pairs available for ion detection, although Geiger-mode avalanche detectors offer some prospect of pushing the detection limits. Ion implantation may have a significant role in fabrication of quantum optical devices where atoms and optical defects are coupled to optical cavities and where the precision in placement of the atom or optical defect is expected to be somewhat more relaxed. Single-ion detection by monitoring changes in the drain current of nano-MOSFETs has been shown to be possible in principle but still requires further development and modelling to understand the optimal gating scheme and the contributions of defects generated in the oxide layers, the channel and other structures with the changes in drain current and IV characteristics observed.

\section{Acknowledgments}

This work was funded by the Australian Research Council through the Centre of Excellence for Quantum Computation and Communications Technology, the Australian Government, the U.S. National Security Agency, and the U.S. Army Research Office under Contract no. W911NF08-1-0527. Financial support was also provided by the EC FP7 FET-proactive NanoICT under Projects nos. MOLOC (215750) and AFSiD (214989), and the Dutch Fundamenteel Onderzoek der Materie FOM.

\section{References}

[1] M. Nielsen and I. Chuang, Quantum Computation and Quantum Information, Cambridge University Press, Cambridge, UK, 2011. 
[2] A. Steane, "Quantum computing," Reports on Progress in Physics, vol. 61, no. 2, pp. 117-173, 1998.

[3] R. Feynman, “There's plenty of room at the bottom," Engineering and Science, vol. 23, no. 5, pp. 22-36, 1960.

[4] R. P. Feynman, "Simulating physics with computers," International Journal of Theoretical Physics, vol. 21, no. 6-7, pp. 467488, 1982.

[5] B. E. Kane, "A silicon-based nuclear spin quantum computer," Nature, vol. 393, no. 6681, pp. 133-137, 1998.

[6] R. Vrijen, E. Yablonovitch, K. Wang et al., "Electron-spinresonance transistors for quantum computing in silicongermanium heterostructures," Physical Review A, vol. 62, no. 1, pp. 012306-012301, 2000.

[7] C. D. Hill, L. C. L. Hollenberg, A. G. Fowler, C. J. Wellard, A. D. Greentree, and H. S. Goan, "Global control and fast solid-state donor electron spin quantum computing," Physical Review B, vol. 72, no. 4, Article ID 045350, pp. 1-9, 2005.

[8] L. C. L. Hollenberg, A. S. Dzurak, C. Wellard et al., "Chargebased quantum computing using single donors in semiconductors," Physical Review B, vol. 69, no. 11, Article ID 113301, pp. 1133011-1133014, 2004.

[9] B. Koiller, X. Hu, and S. Das Sarma, "Exchange in silicon-based quantum computer architecture," Physical Review Letters, vol. 88, no. 2, Article ID 027903, pp. 279031-279034, 2002.

[10] L. C. L. Hollenberg, A. D. Greentree, A. G. Fowler, and C. J. Wellard, "Two-dimensional architectures for donor-based quantum computing," Physical Review B, vol. 74, no. 4, Article ID 045311, 2006.

[11] S. J. Angus, A. J. Ferguson, A. S. Dzurak, and R. G. Clark, "Gate-defined quantum dots in intrinsic silicon," Nano Letters, vol. 7, no. 7, pp. 2051-2055, 2007.

[12] A. Morello, C. C. Escott, H. Huebl et al., "Architecture for high-sensitivity single-shot readout and control of the electron spin of individual donors in silicon," Physical Review B, vol. 80, no. 8, Article ID 081307R, 2009.

[13] K. Y. Tan, K. W. Chan, M. Möttönen et al., "Transport Spectroscopy of single phosphorus donors in a silicon nanoscale transistor," Nano Letters, vol. 10, no. 1, pp. 11-15, 2010.

[14] A. Morello, J. J. Pla, F. A. Zwanenburg et al., "Single-shot readout of an electron spin in silicon," Nature, vol. 467, no. 7316, pp. 687-691, 2010.

[15] T. Shinada, S. Okamoto, T. Kobayashi, and I. Ohdomari, "Enhancing semiconductor device performance using ordered dopant arrays," Nature, vol. 437, no. 7062, pp. 1128-1131, 2005.

[16] K. Bernstein, D. J. Frank, A. E. Gattiker et al., "Highperformance CMOS variability in the $65 \mathrm{~nm}$ regime and beyond," IBM Journal of Research and Development, vol. 50, no. 4-5, pp. 433-449, 2006.

[17] M. Hori, T. Shinada, K. Taira et al., "Performance enhancement of semiconductor devices by control of discrete dopant distribution," Nanotechnology, vol. 20, no. 36, Article ID 365205, 2009.

[18] T. Shinada, T. Kurosawa, H. Nakayama, Y. Zhu, M. Hori, and I. Ohdomari, "A reliable method for the counting and control of single ions for single-dopant controlled devices," Nanotechnology, vol. 19, no. 34, Article ID 345202, 2008.

[19] T. Shinada, M. Hori, K. Taira, T. Endoh, and I. Ohdomari, "Recent advance in single-ion implantation method for singledopant devices," in Proceedings of the 9th International Workshop on Junction Technology, IWJT 2009, pp. 96-99, Kyoto, Japan, June 2009.

[20] M. Pierre, R. Wacquez, X. Jehl, M. Sanquer, M. Vinet, and O. Cueto, "Single-donor ionization energies in a nanoscale
CMOS channel," Nature Nanotechnology, vol. 5, no. 2, pp. 133 $137,2010$.

[21] E. L. Waller and T. Shinada, Eds., "ITRS ERM workshop series: deterministic doping," Berkeley, Calif, USA, 2010 http://www .src.org/calendar/e004100/.

[22] J. R. Rabeau, P. Reichart, G. Tamanyan et al., "Implantation of labelled single nitrogen vacancy centers in diamond using 15N," Applied Physics Letters, vol. 88, no. 2, Article ID 023113, pp. 1-3, 2006.

[23] G. D. Fuchs, V. V. Dobrovitski, R. Hanson et al., "Excited-state spectroscopy using single Spin manipulation in diamond," Physical Review Letters, vol. 101, no. 11, Article ID 117601, 2008.

[24] S. Pezzagna, D. Wildanger, P. Mazarov et al., "Nanoscale engineering and optical addressing of single spins in diamond," Small, vol. 6, no. 19, pp. 2117-2121, 2010.

[25] D. M. Toyli, C. D. Weis, G. D. Fuchs, T. Schenkel, and D. D. Awschalom, "Chip-scale nanofabrication of single spins and spin arrays in diamond," Nano Letters, vol. 10, no. 8, pp. 31683172, 2010.

[26] P. Spinicelli, A. Dréau, L. Rondin et al., "Engineered arrays of nitrogen-vacancy color centers in diamond based on implantation of cn? Molecules through nanoapertures," New Journal of Physics, vol. 13, Article ID 025014, 2011.

[27] H. Huebl, C. D. Nugroho, A. Morello et al., "Electron tunnel rates in a donor-silicon single electron transistor hybrid," Physical Review B, vol. 81, no. 23, Article ID 235318, 2010.

[28] T. Schenkel, A. Persaud, S. J. Park et al., "Solid state quantum computer development in silicon with single ion implantation," Journal of Applied Physics, vol. 94, no. 11, pp. 7017-7024, 2003.

[29] V. Millar, C. I. Pakes, S. Prawer, B. Rout, and D. N. Jamieson, "Thin film resists for registration of single-ion impacts," Nanotechnology, vol. 16, no. 6, pp. 823-826, 2005.

[30] D. N. Jamieson, C. Yang, T. Hopf et al., "Controlled shallow single-ion implantation in silicon using an active substrate for sub- 20-keV ions," Applied Physics Letters, vol. 86, no. 20, Article ID 202101, pp. 1-3, 2005.

[31] J. F. Ziegler, J. P. Biersack, and U. Littmark, SRIM The Stopping and Range of Ions in Solids, Pergamon, New York, NY, USA, 1985.

[32] T. Hopf, Single ion implantation for construction of a quantum computer, Ph.D. thesis, University of Melbourne, 2007.

[33] T. Hopf, C. Yang, S. M. Hearne et al., "Low-noise detection system for the counted implantation of single ions in silicon," IEEE Transactions on Nuclear Science, vol. 55, no. 2, Article ID 4484234, pp. 812-816, 2008.

[34] T. Schenkel, J. A. Liddle, A. Persaud et al., "Electrical activation and electron spin coherence of ultralow dose antimony implants in silicon," Applied Physics Letters, vol. 88, no. 11, Article ID 112101, 2006.

[35] T. Schenkel, C. C. Lo, C. D. Weis, A. Schuh, A. Persaud, and J. Bokor, "Critical issues in the formation of quantum computer test structures by ion implantation," Nuclear Instruments and Methods in Physics Research, Section B, vol. 267, no. 16, pp. 2563-2566, 2009.

[36] C. C. Lo, J. Bokor, T. Schenkel, A. M. Tyryshkin, and S.A. Lyon, "Spin-dependent scattering off neutral antimony donors in 28Si field-effect transistors," Applied Physics Letters, vol. 91, Article ID 242106, 3 pages, 2007.

[37] J. C. McCallum, M. L. Dunn, and E. Gauja, "Ion implantation through thin silicon dioxide layers for Si-based solid-state quantum computer device development," MRS Proceedings, vol. 1074, Article ID 1074-I12-05, 2008. 
[38] J. A. Van Donkelaar, A. D. Greentree, A. D. C. Alves, L. M. Jong, L. C. L. Hollenberg, and D. N. Jamieson, “Top-down pathways to devices with few and single atoms placed to high precision," New Journal of Physics, vol. 12, Article ID 065016, p. 1367, 2010.

[39] H. O. Funsten, S. M. Ritzau, R. W. Harper, and R. Korde, "Response of $100 \%$ internal carrier collection efficiency silicon photodiodes to low-energy ions," IEEE Transactions on Nuclear Science, vol. 48, no. 6, pp. 1785-1789, 2001.

[40] T. Hopf, C. Yang, S. E. Andresen, and D. N. Jamieson, "The response of silicon detectors to low-energy ion implantation," Journal of Physics Condensed Matter, vol. 20, no. 41, Article ID 415205, 2008.

[41] B. C. Johnson, G. C. Tettamanzi, A. D. C. Alves et al., "Drain current modulation in a nanoscale field-effect-transistor channel by single dopant implantation," Applied Physics Letters, vol. 96, no. 26, Article ID 264102, 2010.

[42] A. D. Greentree, J. H. Cole, A. R. Hamilton, and L. C. L. Hollenberg, "Coherent electronic transfer in quantum dot systems using adiabatic passage," Physical Review B, vol. 70, no. 23, Article ID 235317, pp. 1-6, 2004.

[43] R. Rahman, R. P. Muller, J. E. Levy et al., "Coherent electron transport by adiabatic passage in an imperfect donor chain," Physical Review B, vol. 82, no. 15, Article ID 155315, 2010.

[44] Y. P. Song and B. Golding, "Manipulation and decoherence of acceptor states in silicon," EPL, vol. 95, no. 4, Article ID 47004, 2011.

[45] A. M. Tyryshkin, S. A. Lyon, T. Schenkel et al., "Electron spin coherence in Si," Physica E, vol. 35, no. 2, pp. 257-263, 2006.

[46] M. J. Calderón, B. Koiller, and S. D. Sarma, "External field control of donor electron exchange at the $\mathrm{Si} / \mathrm{Si} \mathrm{O}_{2}$ interface," Physical Review B, vol. 75, no. 12, Article ID 125311, 2007.

[47] H. O. Funsten, S. M. Ritzau, R. W. Harper, and R. Korde, "Fundamental limits to detection of low-energy ions using silicon solid-state detectors," Applied Physics Letters, vol. 84, no. 18, pp. 3552-3554, 2004.

[48] H. O. Funsten, S. M. Ritzau, R. W. Harper, J. E. Borovsky, and R. E. Johnson, "Energy loss by keV ions in silicon," Physical Review Letters, vol. 92, no. 21, Article ID 213201, 2004.

[49] A. Akkerman and J. Barak, "New partition factor calculations for evaluating the damage of low energy ions in silicon," IEEE Transactions on Nuclear Science, vol. 53, no. 6, pp. 3667-3674, 2006.

[50] A. Akkerman and J. Barak, "Partitioning to elastic and inelastic processes of the energy deposited by low energy ions in silicon detectors," Nuclear Instruments and Methods in Physics Research, Section B, vol. 260, no. 2, pp. 529-536, 2007.

[51] P. A. Ekstrom, "Triggered-avalanche detection of optical photons," Journal of Applied Physics, vol. 52, no. 11, pp. 6974-6979, 1981.

[52] J. A. Seamons, E. Bielejec, M. S. Carroll, and K. D. Childs, "Room temperature single ion detection with Geiger mode avalanche diode detectors," Applied Physics Letters, vol. 93, no. 4, Article ID 043124, 2008.

[53] E. Bielejec, J. A. Seamons, and M. S. Carroll, "Single ion implantation for single donor devices using Geiger mode detectors," Nanotechnology, vol. 21, no. 8, Article ID 085201, 2010.

[54] J. Meijer, T. Vogel, B. Burchard et al., "Concept of deterministic single ion doping with sub-nm spatial resolution," Applied Physics A, vol. 83, no. 2, pp. 321-327, 2006.

[55] J. L. Hanssen, S. B. Hill, J. Orloff, and J. J. McClelland, "Magneto-optical-trap-based, high brightness ion source for
Use as a nanoscale probe," Nano Letters, vol. 8, no. 9, pp. 28442850, 2008.

[56] W. Schnitzler, N. M. Linke, R. Fickler, J. Meijer, F. SchmidtKaler, and K. Singer, "Deterministic ultracold ion source targeting the Heisenberg limit," Physical Review Letters, vol. 102, no. 7, Article ID 070501, 2009.

[57] T. Schenkel, V. Radmilovic, E. A. Stach, S. J. Park, and A. Persaud, "Formation of a few nanometer wide holes in membranes with a dual beam focused ion beam system," Journal of Vacuum Science and Technology B, vol. 21, no. 6, pp. 2720-2723, 2003.

[58] A. Persaud, K. Ivanova, Y. Sarov et al., "Micromachined piezoresistive proximal probe with integrated bimorph actuator for aligned single ion implantation," Journal of Vacuum Science and Technology B, vol. 24, no. 6, pp. 3148-3151, 2006.

[59] Systems AG Attocube, "Königinstrasse 11a RGB 80539," Munchen, Germany.

[60] S. R. Schofield, N. J. Curson, M. Y. Simmons et al., "Atomically precise placement of single dopants in Si," Physical Review Letters, vol. 91, no. 13, pp. 1361041-1361044, 2003.

[61] M. Fuechsle, S. Mahapatra, F. A. Zwanenburg, M. Friesen, M. A. Eriksson, and M. Y. Simmons, "Spectroscopy of fewelectron single-crystal silicon quantum dots," Nature Nanotechnology, vol. 5, no. 7, pp. 502-505, 2010.

[62] E. Peter, P. Senellart, D. Martrou et al., "Exciton-photon strong-coupling regime for a single quantum dot embedded in a microcavity," Physical Review Letters, vol. 95, no. 6, Article ID 067401, pp. 1-4, 2005.

[63] A. Batra, C. D. Weis, J. Reijonen et al., "Detection of low energy single ion impacts in micron scale transistors at room temperature," Applied Physics Letters, vol. 91, no. 19, Article ID 193502, 2007.

[64] C. D. Weis, A. Schuh, A. Batra et al., "Single atom doping for quantum device development in diamond and silicon," Journal of Vacuum Science and Technology B, vol. 26, no. 6, pp. 2596-2600, 2008.

[65] G. P. Lansbergen, R. Rahman, C. J. Wellard et al., "Gateinduced quantum-confinement transition of a single dopant atom in a silicon FinFET," Nature Physics, vol. 4, no. 8, pp. 656-661, 2008.

[66] “Synopsys, Inc., 700 East Middlefield Road, Mountain,” View, CA 94043. 

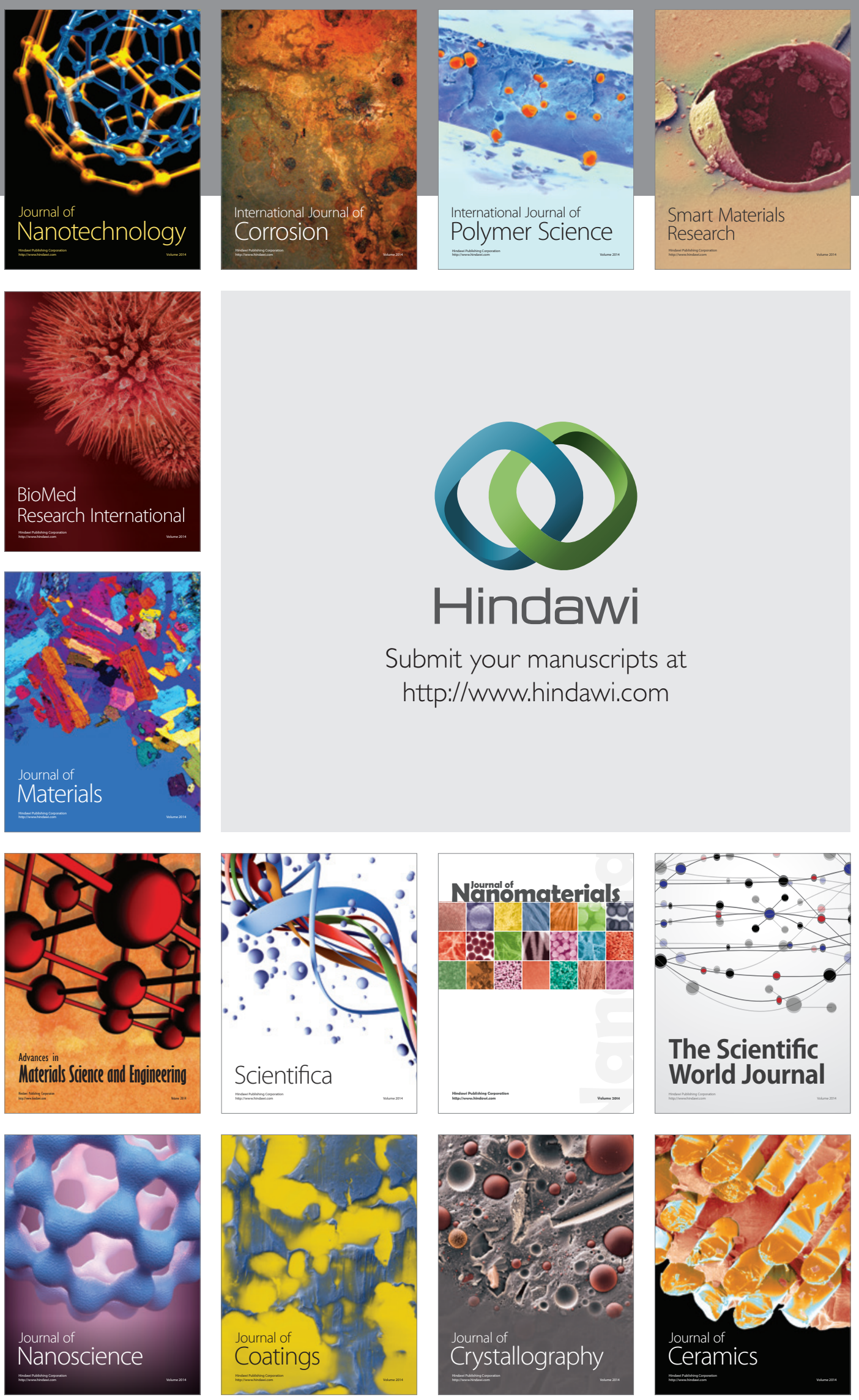

The Scientific World Journal

Submit your manuscripts at

http://www.hindawi.com

\section{World Journal}

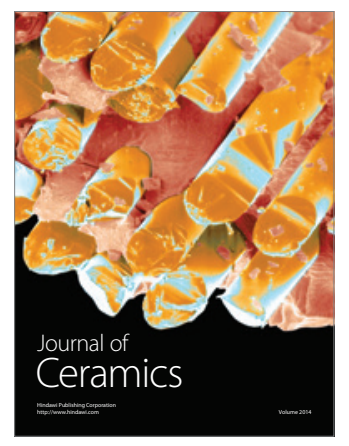

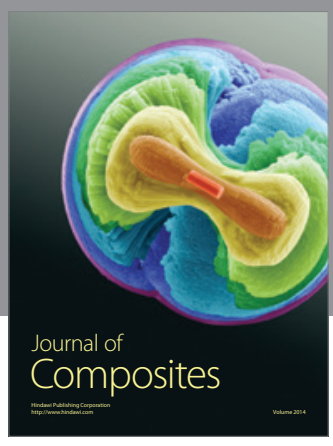
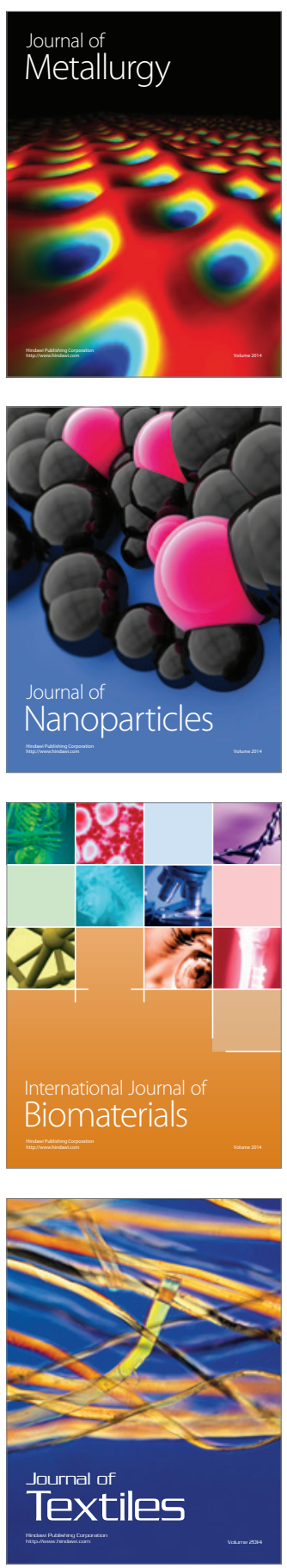